\title{
DESIGN AND FLOW SIMULATION OF TRUNCATED AEROSPIKE
} NOZZLE

\author{
Vinay Kumar Levaka', Srinivasa Reddy $\mathrm{K}^{2}$ \\ ${ }^{1}$ Student, Aerospace dept., Aurora's Scientific and Technological Institute, Hyderabad, Telangana, India \\ ${ }^{2}$ Professor, C.M.R. Engineering College, Hyderabad, Telangana, India
}

\begin{abstract}
Aerospike nozzles are being considered in the development of the Single Stage to Orbit launching vehicles because of their prominent features and altitude compensating characteristics. This paper presents the design of aerospike nozzles using characteristic method in conjunction with streamline function, and performance study through numerical simulation using commercial Computational Fluid Dynamics (CFD) code ANSYS FLUENT. For this purpose nozzles with truncation lengths of $25 \%, 40 \%, 50 \%$ are choosen, because of the thermal and structural complications in the ideal aerospike nozzle. Simulation of the flow is carried out at three different altitude conditions representing Under-expansion, Ideal, and over-expansion conditions of the flow. FLUENT predictions were used to verify the isentropic flow assumption and that the working fluid reached the design exit Mach number. The flow-fields obtained through the numerical simulation are analysed to know the effect of truncation on the performance of aerospike nozzle. Optimum percentage of the truncation is selected by the comparison of nozzles with different lengths of truncation under various altitude parameters. The results show that the flow pattern of the nozzles under different altitude conditions are almost similar. The $40 \%$ truncated nozzle is found to give optimum performance and it has achieved the desired exit Mach number in all the three altitude conditions.
\end{abstract}

Keywords: Aerospike Nozzle, Single Stage to Orbit (SSTO), Linear Aerospike, Truncation and Rocket Nozzle $* * *$

\section{INTRODUCTION}

Ever since jet and rocket propulsion systems have emerged, researchers have invented and implemented many types of nozzles, mainly to increase the thrust performance of nozzles in off-design working conditions. Among these various designs, features of the aerospike nozzle have attracted researchers since mid-1950s. Many theoretical studies of the aerospike nozzle have been carried out in 1960s. In early 1970s, thermal and strength problems of the aerospike nozzle and development of more efficient methods for fabrication of conventional nozzles led to a decline in research activities in this field. Development of the nozzle with the capability of producing optimum amounts of thrust in wide ranges of altitude has been a subject of continuous dedicated efforts within the community of rocket propulsion.

The phenomenon of producing optimum amounts of thrust by a rocket nozzle in off-design conditions is called as altitude compensation. Nozzles with the altitude compensation characteristics are basic feature in realizing the development of Single Stage to Orbit (SSTO) vehicles. Reusable SSTO vehicles offer the promise of reduced launch expenses by eliminating recurring costs associated with hardware replacement inherent in expendable launch systems. The most popular altitude compensating rocket nozzle to date is the aerospike nozzle, the origin of which dates back to Rocketdyne in 1950s.

\subsection{Aerospike nozzle}

An aerospike nozzle has a spike in the center of the nozzle. Aerospike nozzle can be described as an inverted bell nozzle where the flow expands on the outside of the nozzle instead of being completely constrained by the nozzle walls. From the throat, the innermost streamlines of the flow follow the contours of the spike, gradually being turned in the axial direction. Aerospike rocket nozzles are designed for consistent performance over a wide range of ambient pressures.

Traditional converging-diverging nozzles have a single ambient pressure at which the rocket exhaust gases are neither over-expanded nor under-expanded. As the operating conditions move away from the design nozzle pressure ratio (NPR), a shock or an expansion fan will form at the exit plane of the converging-diverging nozzle. These result in reductions in the efficiency of the nozzle. An aerospike nozzle does not have a solid geometry defining the outer limits of the flow path in the supersonic region of the flow. Instead it allows the exhaust gases to expand freely beyond the throat, via the mechanism of a Prandtl-Meyer expansion fan.

\subsection{Advantages}

Smaller nozzle: The truncated spike can be far smaller than a typical bell nozzle for the same performance, as shown below. In addition, a spike can give greater performance for a given length. 


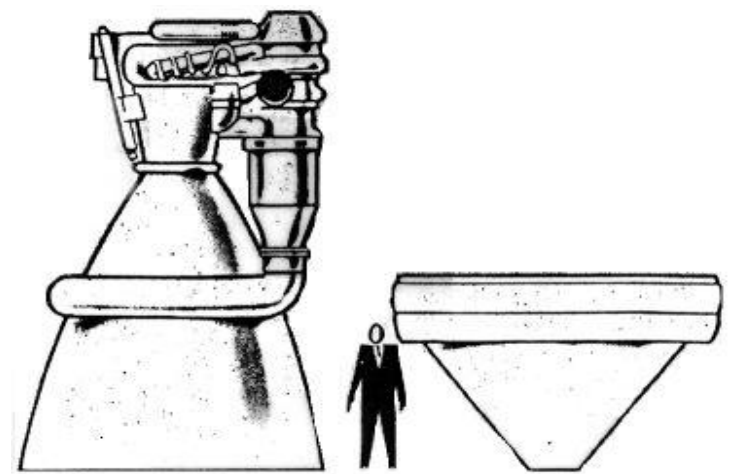

Fig-1: Size comparison of a bell and a plug nozzle

Superior performance: Altitude compensation may result in greater installed performance.

Lower vehicle drag: The aerospike nozzle fills the base portion of the vehicle thereby reducing a type of drag called base drag.

Modular combustion chambers: The linear aerospike engine is made up of these small, easier to develop, less expensive thrusters that give the engine greater versatility.

Thrust vectoring: Because the combustion chambers can be controlled individually, the vehicle can be maneuvered using differential thrust vectoring. This eliminates the need for the heavy gimbals and actuators used to vary the direction of traditional nozzles.

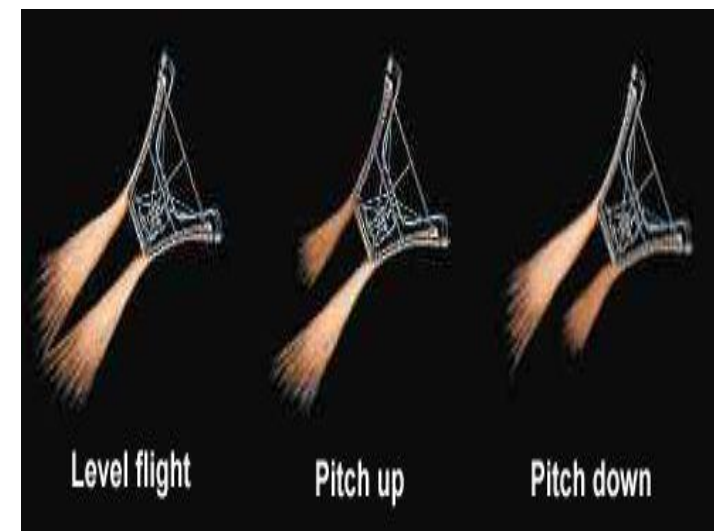

Fig-2: Aerospike thrust vectoring control

Lower vehicle weight: Even though the aerospike tends to be heavier than the bell nozzle, it shares many major structural elements with the vehicle reducing overall weight.

\subsection{Disadvantages}

Cooling: The central spike experiences far greater heat fluxes than does a bell nozzle. This problem can be addressed by truncating the spike to reduce the exposed area and by passing cold cryogenically-cooled fuel through the spike. The secondary flow also helps to cool the centerbody. Manufacturing: The aerospike is more complex and difficult to manufacture than the bell nozzle. As a result, it is more costly.
Flight experience: No aerospike engine has ever flown in a rocket application. As a result, little flight design experience has been gained.

\section{DESIGN METHODOLOGY}

Design of the aerospike nozzle mainly refers to the design of the central spike and the determination of angle of the primary nozzle. Method of characteristics in conjunction with the streamline conditions of A.H.Shapiro is used for the design of aerospike nozzle contour. A point on the characteristic line where it satisfies streamline condition will be the point on spike contour. Since the method of characteristics solution is based upon a start line slightly greater than Mach number equal to one, it is apparent that the complete supersonic flow field and the nozzle performance is governed by this parameter.

The gas expansion process in the flow field of the plug nozzle is assumed to be isentropic, adiabatic, and frictionless. The method of characteristics is logically and physically applicable for determining pertinent parameters throughout the flow field of a supersonic isentropic plug nozzle. Expansion process is determined by the PrandtlMeyer expansion function, which follows the below equation

$v=\left(\frac{\gamma+1}{\gamma-1}\right)^{\frac{1}{2}} \tan ^{-1}\left[\frac{\gamma-1}{\gamma+1}\left(M^{2}-1\right)\right]^{\frac{1}{2}}-\tan ^{-1}\left(M^{2}-1\right)^{\frac{1}{2}}$

Because of the aerospike nozzle geometry, the physical nozzle throat area is not normal to the engine centerline but is inclined by a specific angle. Because of the nozzle contour geometry, the combustion products are accelerated differently such that an axisymmetric sonic line shape, even with regard to the nozzle throat centerline, is not formed. The flow direction at the throat is set at an angle equal to the Prandtl-Meyer expansion angle associated with the userdefined exit Mach number. The Prandtl-Meyer expansion fan is centered at the tip of the cowl and its location is userdefined. Fig-3 shows the schematic of the aerospike nozzle displaying the characteristic lines representing PrandtlMeyer expansion waves.

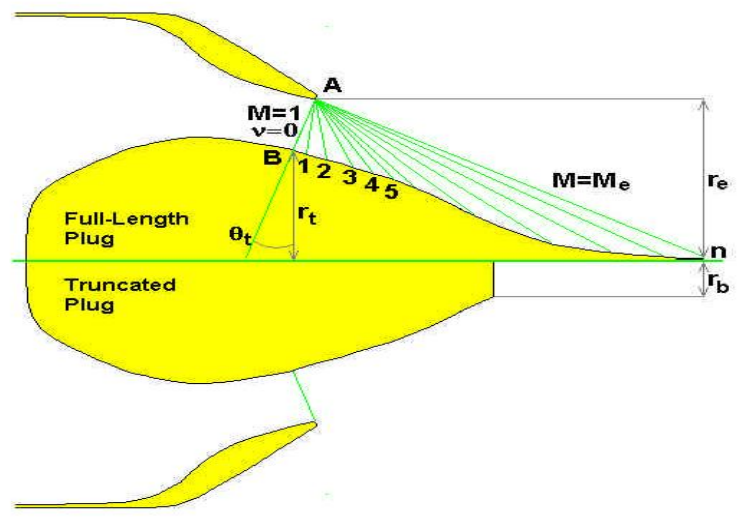

Fig-3: Schematic of the axisymmetric aerospike nozzle 
The technique used in this paper to calculate the contours of the aerospike nozzles are similar to the technique outlined by Lee and Thompson, 1964 [4]. For the traditional aerospike nozzle, the technique used in this paper defines the location of the ends of the throat and sets the flow direction at the throat equal to the Prandtl-Meyer expansion angle associated with the desired exit Mach number. Unlike the technique outlined by Lee and Thompson [4], the calculations used in this paper step forward through the expansion fan by a user-defined Prandtl-Meyer expansion angle increment. The intersection of the characteristics emanating from the expansion point on one end of the throat and the Stream Function originating from the last point calculated on the nozzle's contour define the nozzle's contour. The Prandtl-Meyer expansion fan is stepped through until the Mach number along the characteristic being analyzed is greater than or equal to the desired userdefined exit Mach number, in which case, the intersection of the Stream Function and characteristic signify the location of the last point on the nozzle's contour. The geometry of an aerospike nozzle and the parameters involved can be seen from Fig-4.

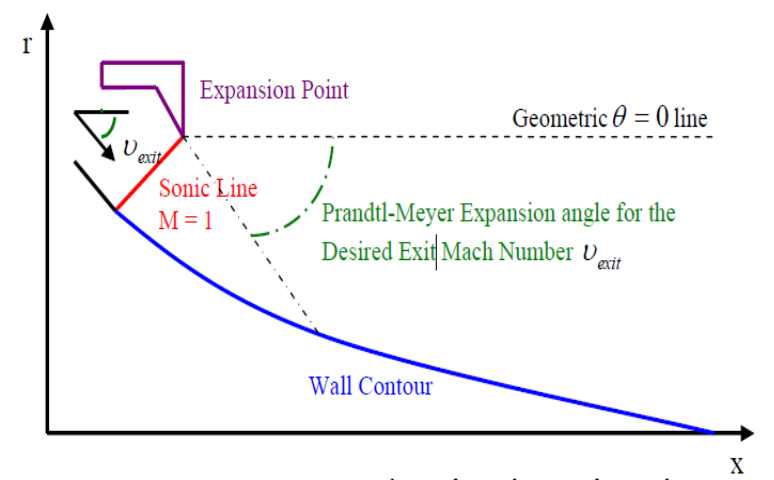

Fig-4: Geometry of an aerospike nozzle

This method becomes more accurate as the number of characteristics used in the calculation increases, which means choosing less value for the change in Prandtl-Meyer angle. The increased number of characteristics also results in a smoother contour.

\subsection{Mach Number Calculation}

The work presented in this paper uses the Bisection Method to calculate the Mach number associated with each flow field point's Prandtl-Meyer expansion angle. The program sets the lower limit of the range to a Mach number of 1 and the upper limit to a 100 times the desired exit Mach number. The program then calculates an initial guess Mach number by averaging the range's limits.

$$
M a_{\text {guess }}=\frac{M a_{\text {upper }}+M a_{\text {lower }}}{2}
$$

Calculate the Prandtl-Meyer expansion angle of the guess Mach number using Prandtl-Meyer expansion function and compares it to the Prandtl-Meyer expansion angle of the point. If the Prandtl-Meyer expansion angle of the guess
Mach number is greater than the point's Prandtl- Meyer expansion angle, the program sets the upper limit of the range equal to the guess Mach number. If the Prandtl-Meyer expansion angle of the guess Mach number is less than the Prandtl-Meyer expansion angle of the point, set the lower limit of the range equal to the guess Mach number. If the difference between the Prandtl-Meyer expansion angle of the guess Mach number and the point's Prandtl-Meyer expansion angle is greater than $\operatorname{abs}\left(1 \mathrm{e}^{-10}\right)$, recalculate a new guess Mach number using the new range limits. If the difference in Prandtl-Meyer expansion angles is less than $\operatorname{abs}\left(1 \mathrm{e}^{-10}\right)$, the guess Mach number will be the point's Mach number.

\section{NUMERICAL SIMULATION}

This section describes numerical modeling and analysis of external flow of the aerospike nozzle with different plug shapes using a commercial CFD code ANSYS FLUENT. The objective of the analysis is comparison of flow patterns produced by aerospike nozzles with different plug shapes. Numerical modeling also helps us to validate the design of the Aerspike nozzle by comparing the expected Mach number with the Mach number obtained from the numerical simulation

\subsection{Geometry}

Using the design theory mentioned in the previous section Aerospike nozzle contours with the nozzle length percentages of 25,40 , and 50 are designed. The parameters used as input for the determination of the contour coordinates are as follows

Expected exhaust Mach number: $\mathrm{Me}=3$

Propellant: Ethanol-Oxygen, $\gamma=1.21$

Throat Radius: $r_{t}=0.0508 \mathrm{~m}=2$ inch

Change in Prandtl-meyer angle $\Delta v=0.005$

Truncation values are taken in accordance with considerations regarding the thermal and structural capabilities of the nozzle. But the increased amounts of truncation leads to the larger base radius which in turn leads to the larger Throat radius, so the design uses constant throat radius. The Co-ordinates of the expansion points and the throat angle which is equal to the $v_{\max }$ are calculated using the Prandtl-Meyer equation.

Expansion point: (1.778, 13.3099)

Throat Angle: $\theta_{\mathrm{t}}=62.7508^{\circ}$ 


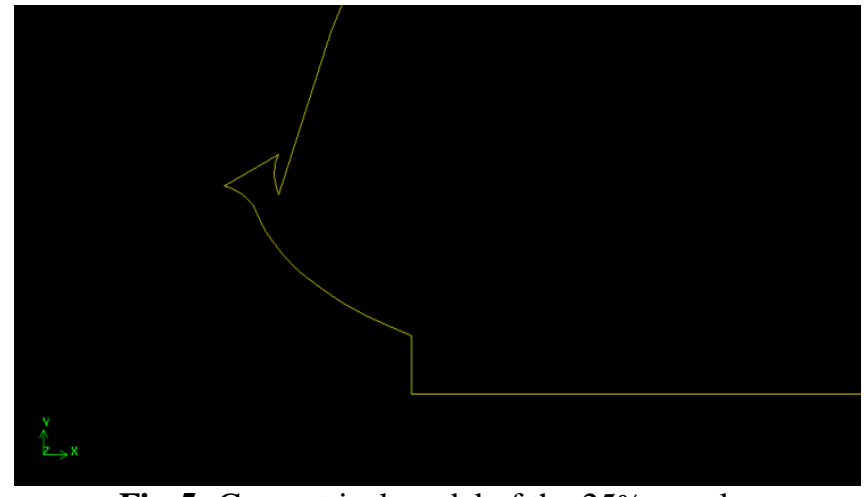

Fig-5: Geometrical model of the $25 \%$ nozzle

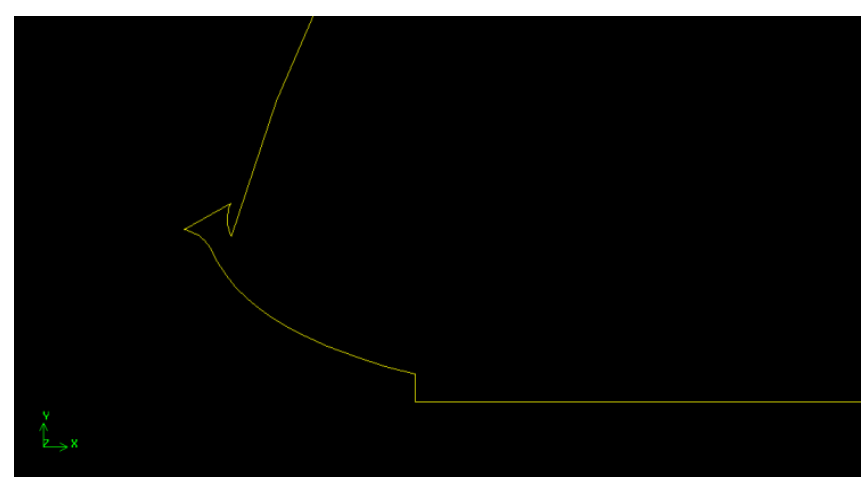

Fig-6: Geometrical model of the $40 \%$ nozzle

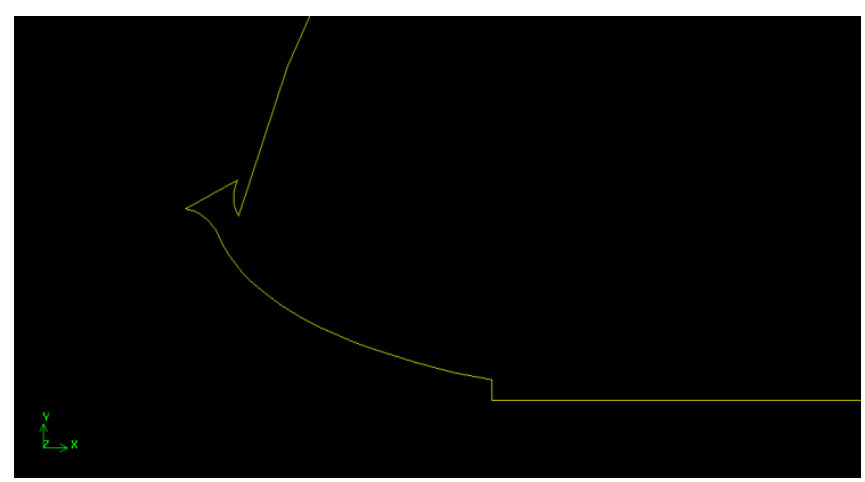

Fig-7: Geometrical model of the 50\% nozzle

Using the co-ordinates of expansion point, nozzle contour co-ordinates, and Throat angle that are calculated, geometrical models are developed and meshed using the GAMBIT, a commercial modeling and meshing tool. Coordinate data obtained from the design calculations is to create the contour surface of the nozzle. The primary nozzle is created by using the two geometrical arcs approximating the convergent section at the throat.

\subsection{Mesh}

The solution domain, in all the cases, is discretized using a structured grid of quadrilateral cells. As the geometric variations in regions surrounding to the nozzle surface make it impossible to generate a structured grid with acceptable quality, domain has been divide into two different faces. Different geometries of the truncated aerospike nozzles cause the solution domain to have different number of cells. Total number of grid cells for the $25 \%, 40 \%$, and $50 \%$ cases are 6566,7388 , and 7241 , respectively.

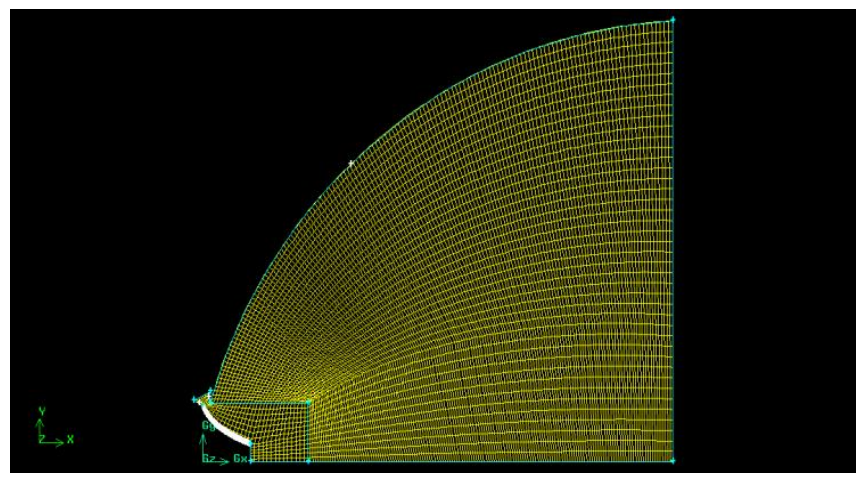

Fig-8: Grid for analysis of $25 \%$ nozzle

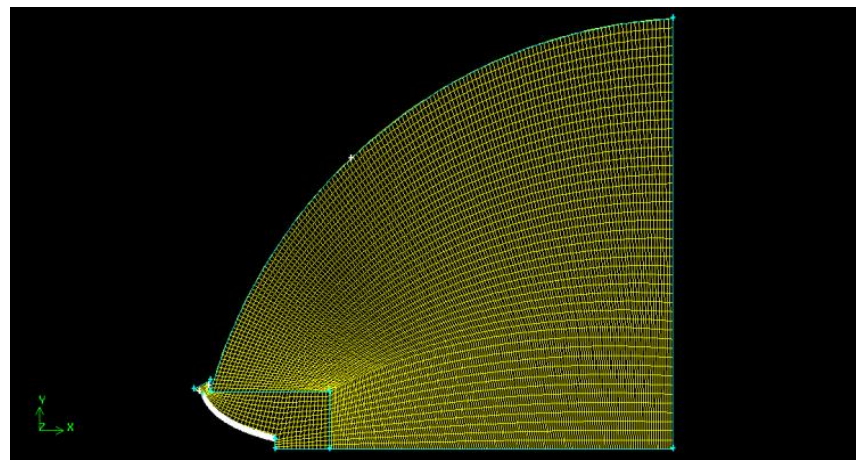

Fig-9: Grid for analysis of $40 \%$ nozzle

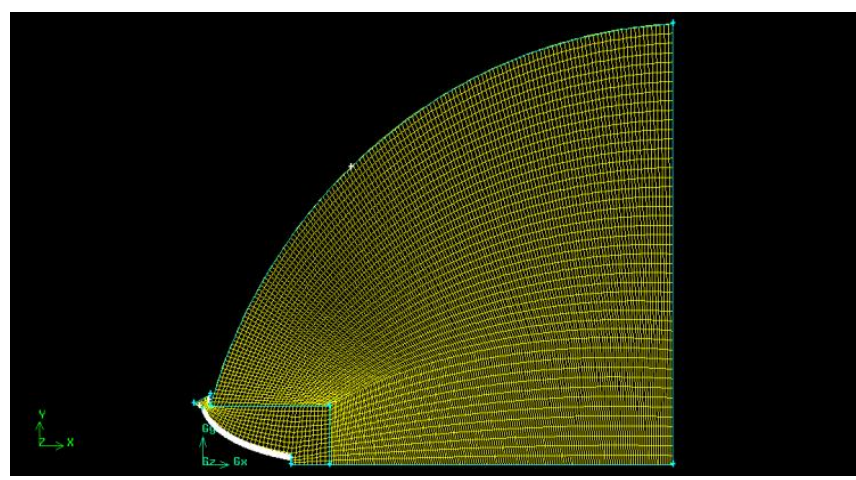

Fig-10: Grid for analysis of 50\% nozzle

\subsection{Boundary Conditions}

The condition implied at the inlet of the convergent section is inlet with specified mass flow, with the following boundary values, these values are taken from the Reference [5]

Mass flow rate $(\mathrm{m})=3.25757 \mathrm{~kg} / \mathrm{s}$

Temperature $(\mathrm{T})=1577.826 \mathrm{~K}$

Pressure $(\mathrm{P})=2045430 \mathrm{~N} / \mathrm{m}^{2}$ 


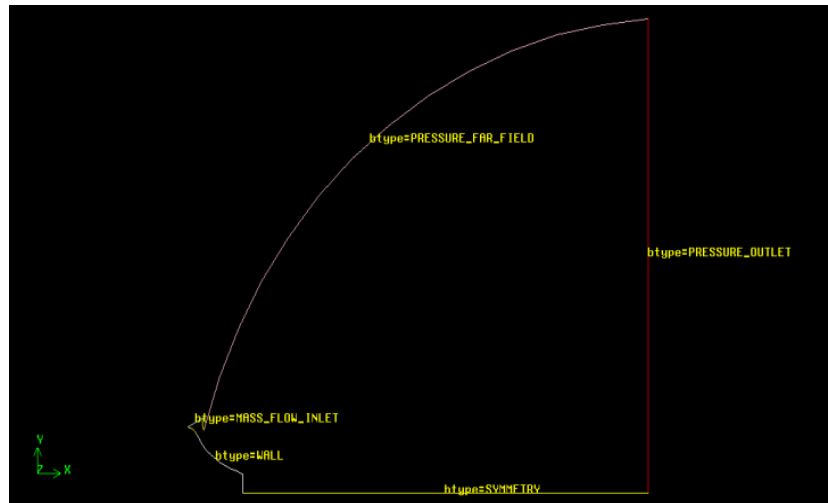

Fig-11 Solution domain and boundary conditions

Boundary values are calculated using the nozzle design parameters mentioned below

Chamber Pressure: P1 = 2067857 N/m²

Design Altitude: $\mathrm{h}=3657.6 \mathrm{~m}$

Mass Flow: $\mathrm{m}=3.25758 \mathrm{~kg} / \mathrm{s}$

Three different cases of atmospheric conditions (Patm/Pdes) have been analyzed, which correspond to different working conditions (including over-expansion, optimum and underexpansion). Boundary values imposed at pressure farfield and pressure outlet boundaries in the 3 cases are presented in Table-1. Apart from case 2, which corresponds to the nozzles' optimum working condition, the other cases have been chosen hypothetically to test the effect of truncation on nozzle performance. Supersonic Mach numbers of external flow has been selected for all cases in order to facilitate convergence criteria. Atmospheric conditions at the design altitudes are given as input pressure and temperature at farfield and pressure outlet conditions.

Table-1: Values imposed at farfield boundaries

\begin{tabular}{|l|l|l|l|l|l|}
\hline CASE & $\mathbf{P}_{\text {atm }} / \mathbf{P}_{\text {des }}$ & $\begin{array}{l}\mathbf{P} \\
\left(\mathbf{N} / \mathbf{m}^{2}\right)\end{array}$ & $\begin{array}{l}\text { Exit } \\
\text { Mach }\end{array}$ & $\begin{array}{l}\text { Temp. at } \\
\text { Farfield } \\
(\mathbf{K})\end{array}$ & $\begin{array}{l}\text { Outle } \\
\mathbf{t} \\
\text { Temp } \\
\mathbf{( K )}\end{array}$ \\
\hline 1 & 1.57 & 101325 & 1.5 & 288.15 & 350 \\
\hline 2 & 1 & 64434 & 3 & 264.37 & 480 \\
\hline 3 & 0.10 & 6410 & 3 & 216.61 & 420 \\
\hline
\end{tabular}

\subsection{Initial Conditions}

Numerical values of flow variables vary greatly in different regions of the solution domain in analysis of a nozzle with external flow. For example, while values close to stagnation properties prevail at the inlet of the convergent section, the external flow might involve substantially lower pressures and higher (even supersonic) Mach numbers. In such circumstances, initialization of a flow variable with a constant value throughout the entire domain can make convergence difficult or sometimes impossible. To deal with this problem, properties at nozzle inlet, throat and exhaust surface, which can be roughly estimated using one dimensional isentropic flow relations, have been used to define custom field functions describing initial values of axial velocity, pressure and temperature in regions surrounding the nozzle.

\subsection{Analysis Features}

The nozzle contours were built on the assumption of inviscid, irrotational, isentropic flow. So In CFD Simulation the fluid is considered as inviscid. The isentropic assumption, which implies irrotationality, was achieved by assigning the specific heat at constant pressure as a constant property of the working fluid. Combustion products have been assumed to behave as a compressible ideal gas $(\mathrm{P}=\rho \mathrm{RT})$. The coupled implicit method has been used for solution of the four governing equations (continuity, conservation of momentum in longitudinal and radial directions, and conservation of energy), considering severe compressibility effects existing in the solution domain. Fluxes of convected variables at cell walls are approximated by the first order upwind scheme. Courant's number has been set to 0.5 for all the cases. This criterion has been posed for convergence. One is reduction of the global residual of solution of all governing equations to the order of $10^{-5}$, and the other is establishment of mass balance between inlet, far-field and outlet boundaries, which is checked by integration of mass flow through the mentioned boundaries at each iteration. In all cases, the solution process has been continued until both criteria have been satisfied.

\section{RESULTS AND DISCUSSIONS}

Total study is divided into three different cases which represent to under-expansion, ideal/ designed conditions and over-expansion conditions.

Exhaust flow of the aerospike nozzle is characterized by formation of a series of expansion waves, which originate from the upper lip of the convergent section. Since the exhaust flow is not bounded by a solid wall, these expansion waves can adjust their intensity and domain to match the exhaust flow with the external flow, which gives an advantage of the altitude compensation in contrast to the conventional nozzle.

\subsection{Over Expansion Conditions}

This case corresponds to the simulation of $25 \%, 40 \%$, and $50 \%$ nozzles in the over-expansion conditions where the pressure ratio $\mathrm{P}_{\mathrm{atm}} / \mathrm{P}_{\mathrm{des}}=1.57$. In this case we can observe that the expansion waves originating from the upper lip of the convergent primary nozzle extend even after the surface of the nozzle meeting at the midpoint of the base, and the secondary expansion can be observed at the tip of the truncated portions. These expansion waves continue their way in spite of the larger amounts of the compression which leads to the over-expansion conditions. The effect of overexpansion can be clearly seen in Fig-12(b), 12(d), and 12(f) showing path-lines of the exhaust flow in the region before 
the stagnation point. After the stagnation point the exhaust gases flow parallel to the axis of symmetry. The stagnation point in all three nozzles, with varying truncation percentages, is nearer to the surface of the truncation base reducing the area of recirculation region where the two vortices are formed. This phenomenon can be clearly observed in the above mentioned figures.

An oblique shock can be seen in this case, which can be observed in Fig-12(a), 12(c), and 12(e) showing the Mach number contours, nearer to the surfaces surrounding the upper lip of the primary nozzle. These waves are characterized by high velocities and expansion. But in all the cases the effect of the shock waves on the exhaust flow is in negligible range. In comparison between all the three nozzles, it is observed that effect of shock waves is more in the $25 \%$ nozzle.

In the Fig-12(a), 12(c), and 12(e) showing the contours of Mach number it is observed that the velocity of the flow in all the cases is within the expected range. Velocity of flow is gradually increased to give the optimum Mach number at the end of the nozzle after the stagnation point. Mach number of the exhaust flow of the three cases are obtained as 2.6 in $25 \%$ nozzle, 3.15 Mach in $40 \%$ nozzle, 3.2 Mach in the $50 \%$ nozzle.

\subsection{Ideal Conditions}

In this case discussion is about the results of simulation of aerospike nozzle with varying truncation amounts at design altitude.

At this condition the flow density pattern is same to that of the previous case but the flow keeps on diverging even after the stagnation point. But this divergence is contained by the surrounding domain. From the Fig-13(b), 13(d), and 13(f) showing the path-lines colored by density it is observed that expansion characterized by decrease in density occurs at the tip of the primary nozzle and again at the tip of the truncated portion of the nozzle. This expansion is followed by the compression of exhaust gases by the surrounding domain. From the Fig-13 the position of the stagnation point and the recirculation area forming two vortices at the base of truncated nozzle can be observed. And the expansion waves are extended even after the end of the nozzle surface. But from the contours of Mach number shown in the Fig-13(a), 13(c), and 13(e) it is observed that velocity of the exhaust flow is gradually increasing even during the compression phase reaching the maximum Mach number of 3.2, 3.7 and 3.5 Mach for $25 \%, 40 \%$ and $50 \%$ nozzles respectively at the exit of the nozzle.

\subsection{Under Expansion Condition}

In this case the expansion waves originated from the upper lip of the primary nozzle face the truncated portion of the plug. The flow facing the truncation first encounters a sharp expansion, then by continuing its way to the centre of the plug base. From this point flow passes through compression by the atmosphere and the flow meets at the stagnation point where the flow properties are nearer to the ideal flow conditions. This phenomenon is due to the formation of two symmetric vortices in the base of the plug, which counteract the effect of each other at two locations, one of which is located at the center of the plug base, where the stagnation conditions prevail. This can be noticed in the Fig-14(b), 14(d), and 14(f) showing the pathlines.

In this case it is clearly visible that the series of expansion waves started at the upper lip of the primary nozzle got compressed by the atmosphere; this compression is characterized by increase in the density and reduction in the velocity of the exhaust flow. Effect of these expansion and compression waves on pathlines is such that the exhaust flow leaves the exit surface straight after the point of stagnation the exhaust gases flow parallel to the axis leaving no residuals, thus producing a great deal of thrust. This can be observed in the Fig-14(b), 14(d), and 14(f) showing the pathlines colored by density.

From the contours of Mach number in Fig-14(a), 14(c), and 14(e) we can observe that velocity of the flow during the expansion after leaving the primary nozzle is high and this velocity is later reduced by the compression of flow achieving a Mach number of 1.5, 3.5, and 1.72 at the nozzle exit for $25 \%, 40 \%$, and $50 \%$ nozzles respectively.

\subsection{Comparison of Results}

In all the cases exhaust flow of the aerospike nozzle is characterized by formation of a series of expansion waves, which originate from the upper lip of the convergent section. Since the exhaust flow is not bounded by a solid wall, these expansion waves can adjust their intensity and domain to match the exhaust flow with the external flow, which gives an advantage of the altitude compensation in contrast to the conventional nozzle.

It should be pointed out that regardless of the amount of truncation and the extent of the plug base area, the flow parameter distribution pattern is the same. Figures showing pathlines clearly show the above-mentioned process in form of path-lines colored by density for different plug shapes. It should also be noted that in spite of existence of rotational flow at the base area, path-lines will continue their way parallel to the axis of the plug after the longitudinal position corresponding to the end of a virtual ideal plug, even for the $25 \%$ truncated aerospike nozzle. In order to understand the concept of thrust delivery by different truncated aerospike nozzles in under-expansion conditions, design conditions and in over-expansion conditions, it is necessary to approach the thrust components differently. Dividing thrust into the three following components, explains this phenomenon more clearly

1) Thrust produced by the nozzle convergent section

2) Thrust produced by the plug surface

3) Thrust produced by the plug base

In under-expansion conditions, when the plug is truncated, its lateral area decreases. Therefore the pressure thrust produced by the plug reduces. On the other hand, thrust 
generated by the base region increases because of the increase of the base area. These two effects compensate each other, and the total nozzle thrust becomes almost the same for different nozzle truncation. This effect can be seen most clearly for the $25 \%$ plug.

But in over-expansion conditions, the situation is totally different. In these conditions, as the nozzle length becomes shorter, hence decreasing the plug area, thrust produced by the plug still decreases, while as the atmosphere pressure is higher than the exhaust pressure thrust produced by the base pressure would have a negative value. So by increasing truncation, the negative value of base thrust will increase, hence decreasing total thrust in over-expansion conditions. It can be concluded that for the $25 \%$ plug, total thrust is lowest. At low altitudes (i.e., over-expansion conditions) base pressure linearly increases as atmospheric pressure increases. At high altitudes, pressure at the base remains constant despite variation of altitude. As the altitude increases, atmospheric pressure decreases and the difference between base pressure and atmospheric pressure increases, hence increasing the base thrust.

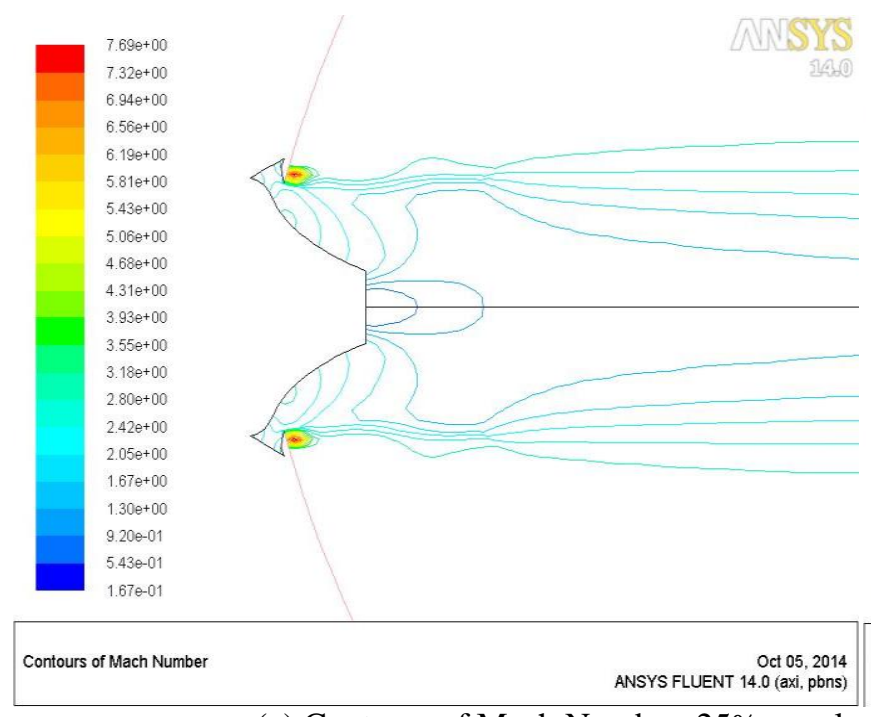

(a) Contours of Mach Number, $25 \%$ nozzle

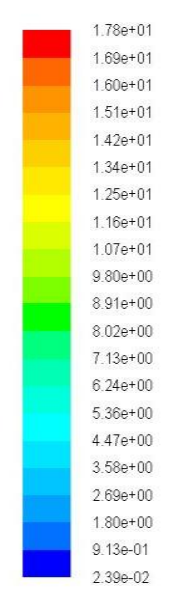

Pathlines Colored by Density ( $\mathrm{kg} / \mathrm{m} 3)$

(b) Pathlines, $25 \%$ nozzle

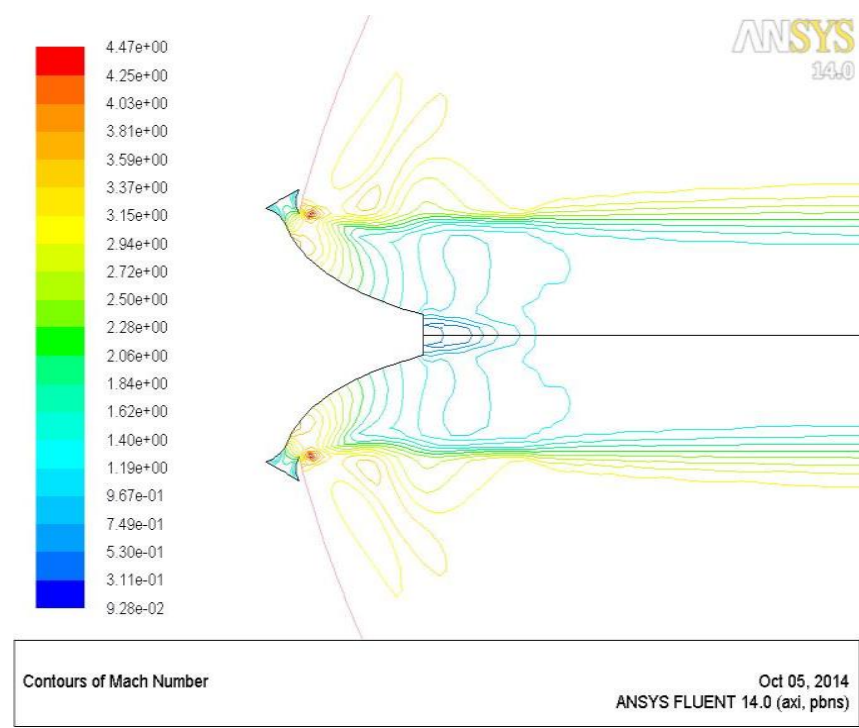

(c) Contours of Mach Number, $40 \%$ nozzle

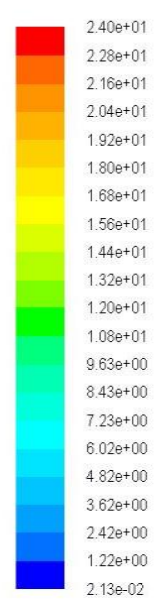

Pathlines Colored by Density $(\mathrm{kg} / \mathrm{m} 3)$

(d) Pathlines, $40 \%$ nozzle
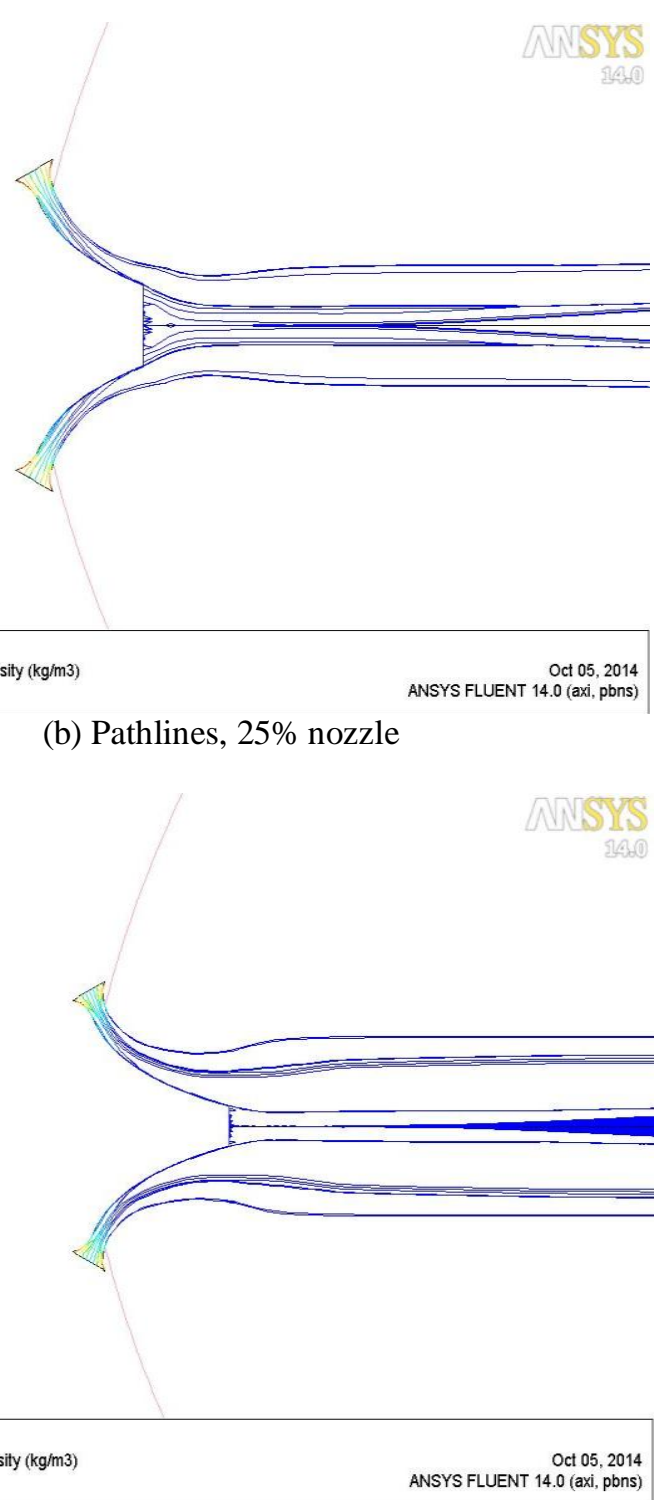

(n) 

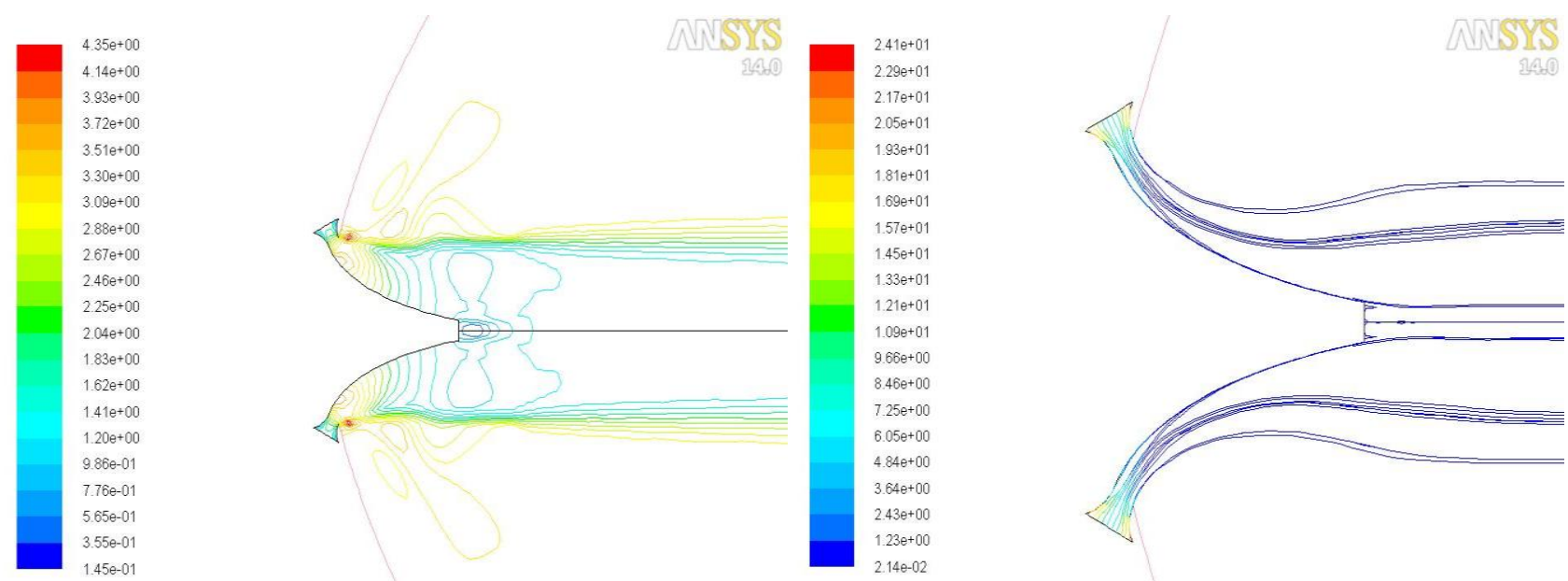

Contours of Mach Number

(f) Pathlines, 50\% nozzle

Fig-12: Flow pattern of the ideal and truncated aerospike nozzles in over-expansion conditions $($ Patm/Pdes $=1.57)$

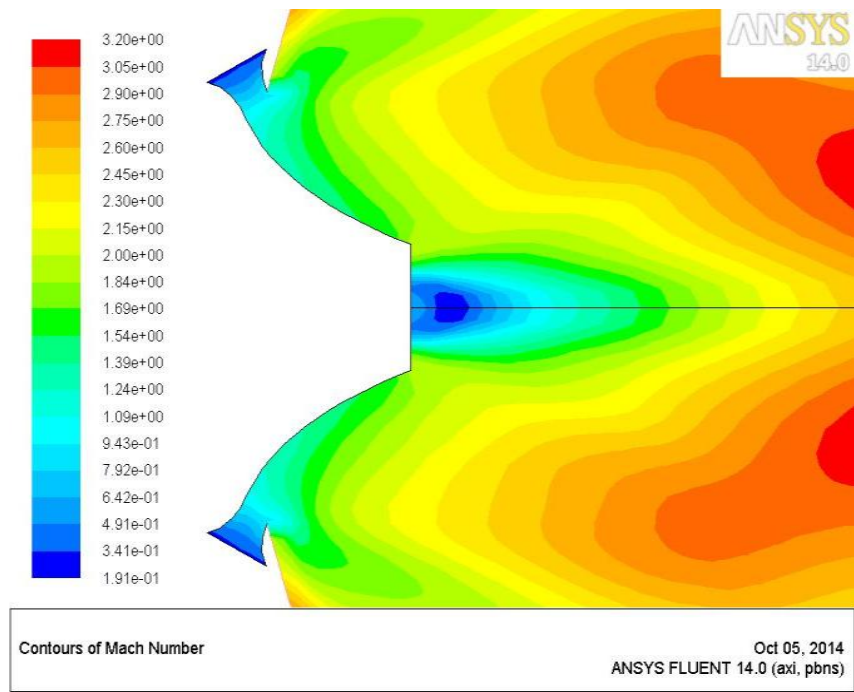

(a) Contours of Mach Number, $25 \%$ nozzle

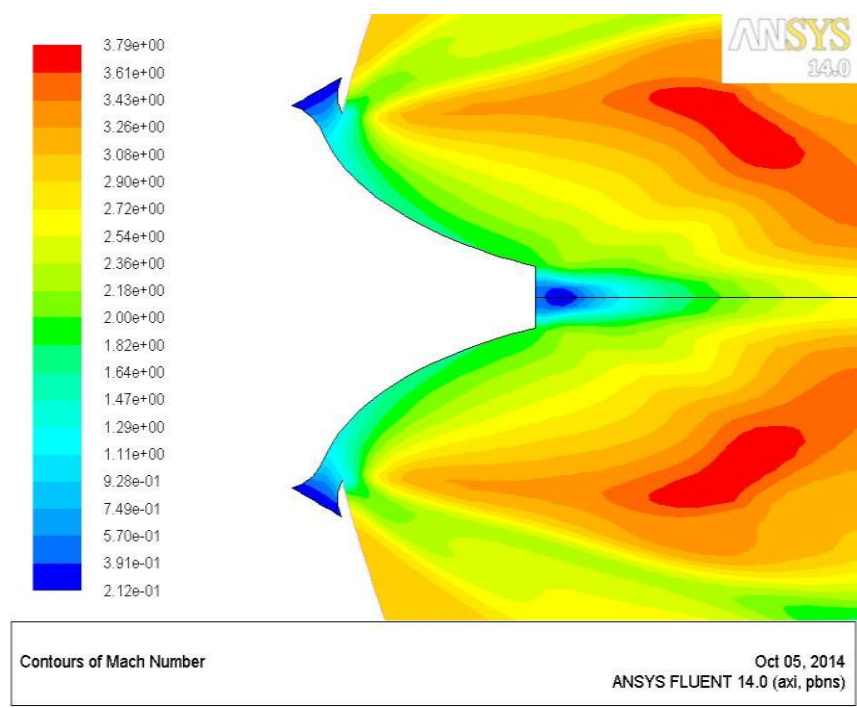

(c) Contours of Mach Number, $40 \%$ nozzle

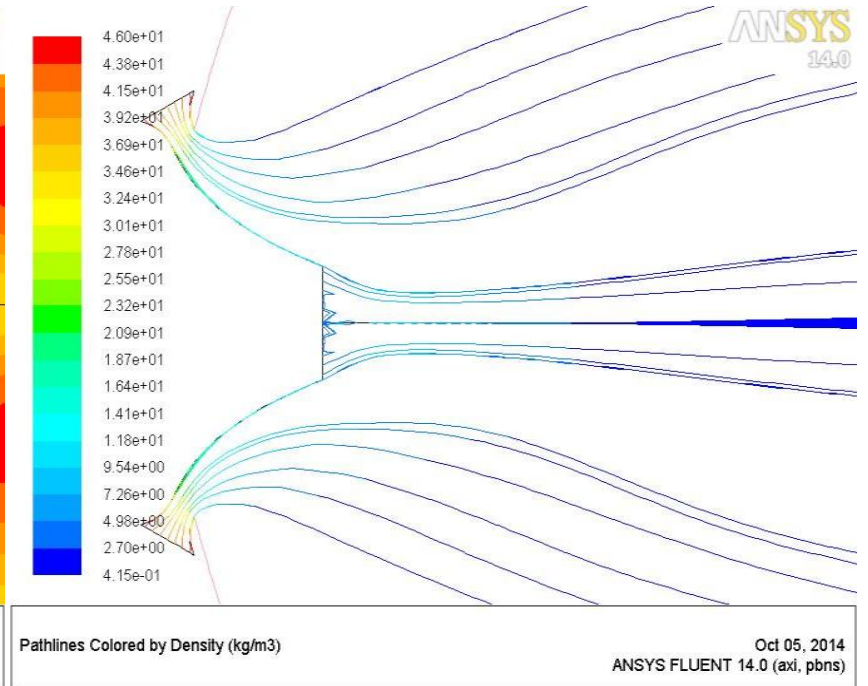

(b) Pathlines, $25 \%$ nozzle

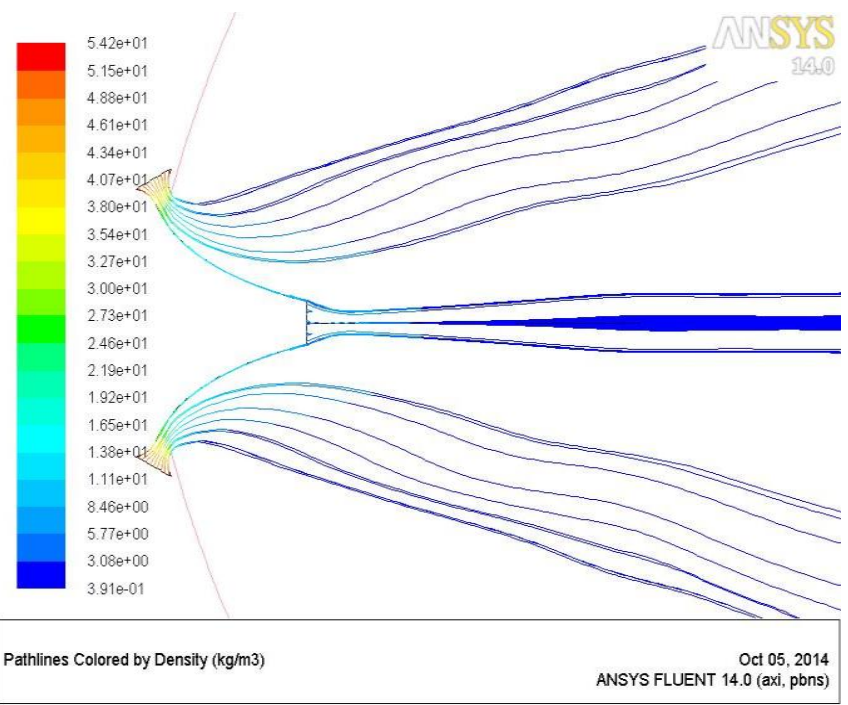

(d) Pathlines, $40 \%$ nozzle 


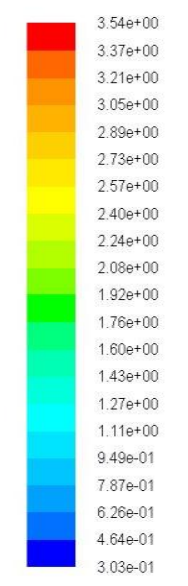

Contours of Mach Number

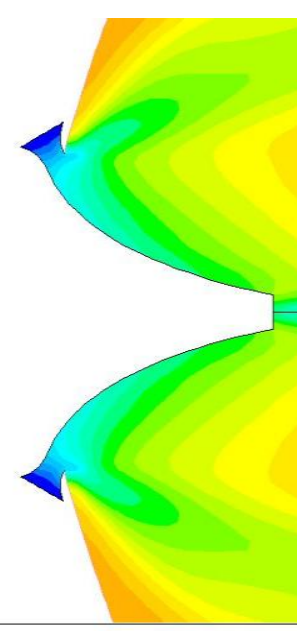

Oct 05, 2014
ANSYS FLUENT 14.0 (axi, pbns)

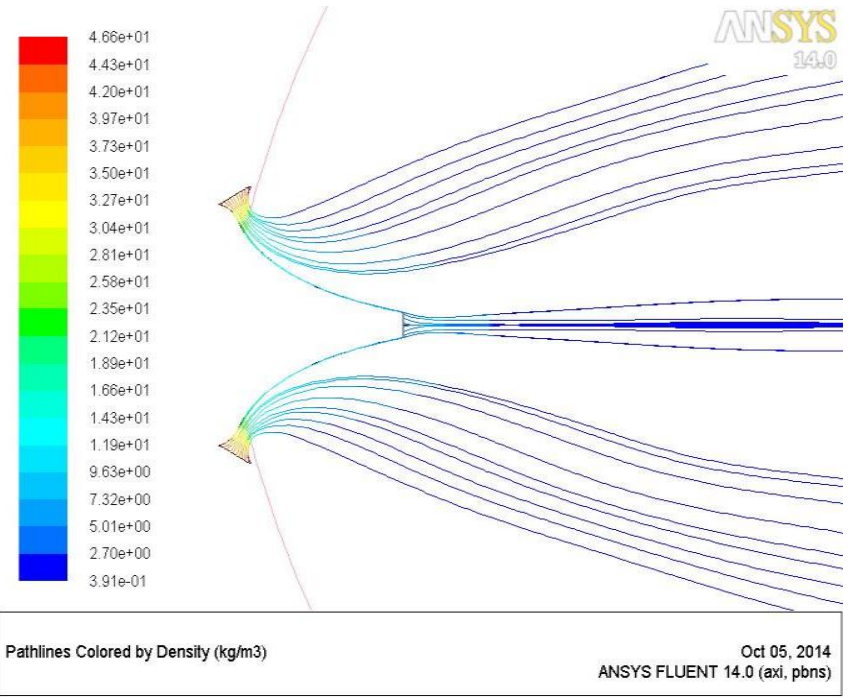

(f) Pathlines, $50 \%$ nozzle

Fig-13: Flow pattern of the ideal and truncated aerospike nozzles in design conditions (Patm/Pdes $=1.00)$
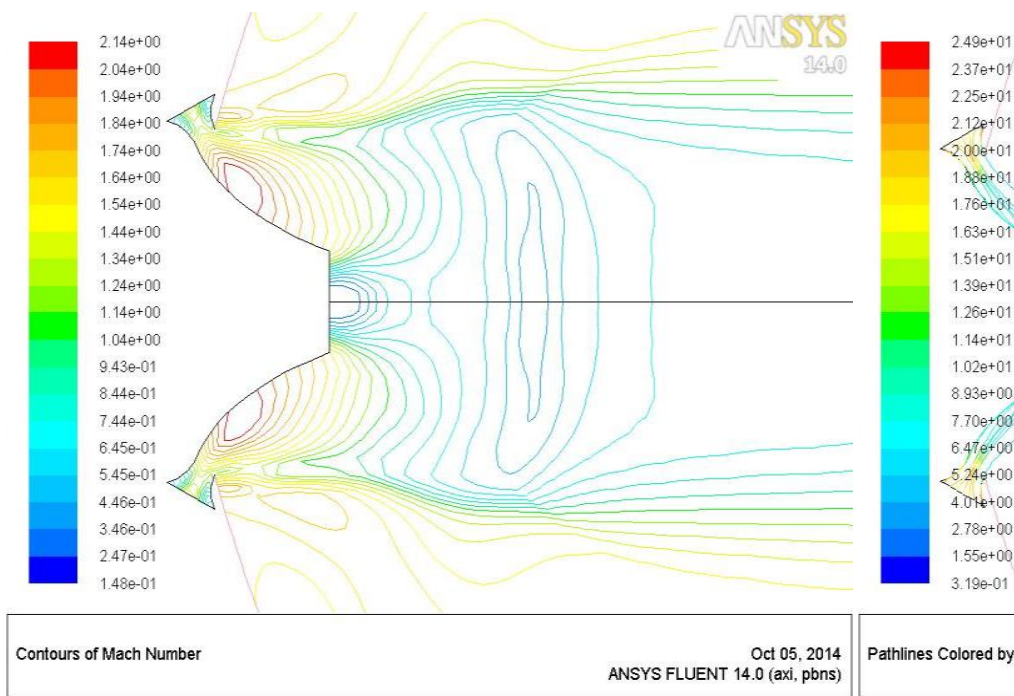

Pathlines Colored by Density $(\mathrm{kg} / \mathrm{m} 3)$

(a) Contours of Mach Number, $25 \%$ nozzle

(b) Pathlines, 25\% nozzle

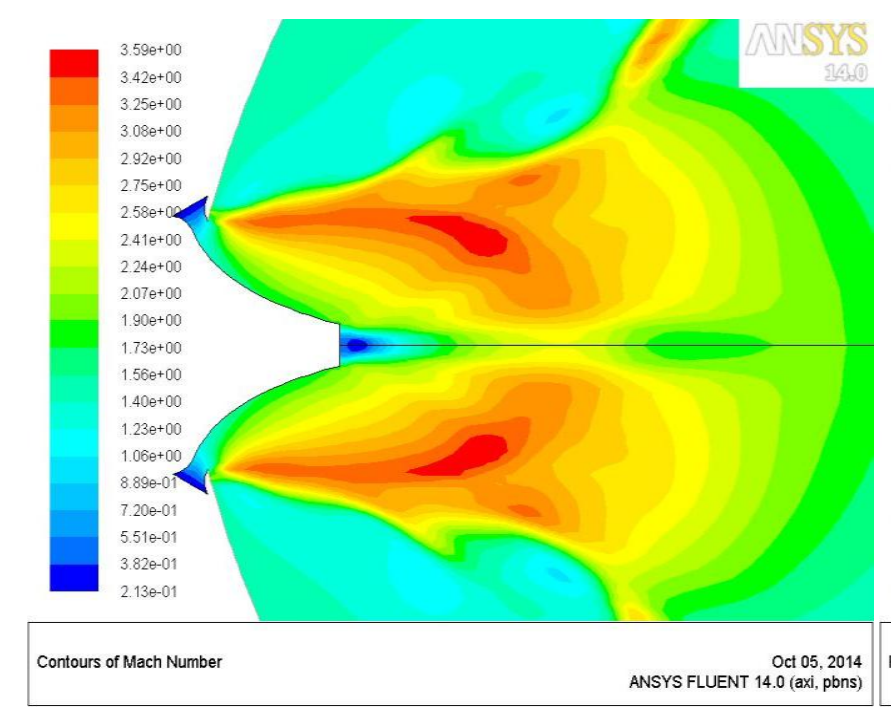

(c) Contours of Mach Number, $40 \%$ nozzle

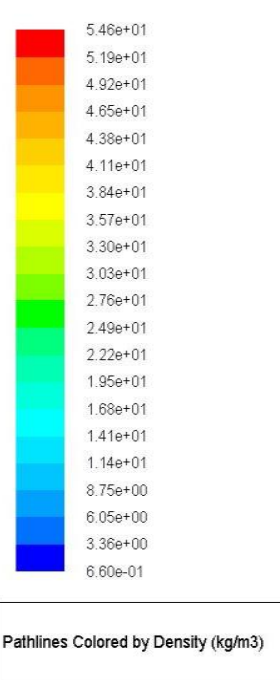

(d) Pathlines, $40 \%$ nozzle

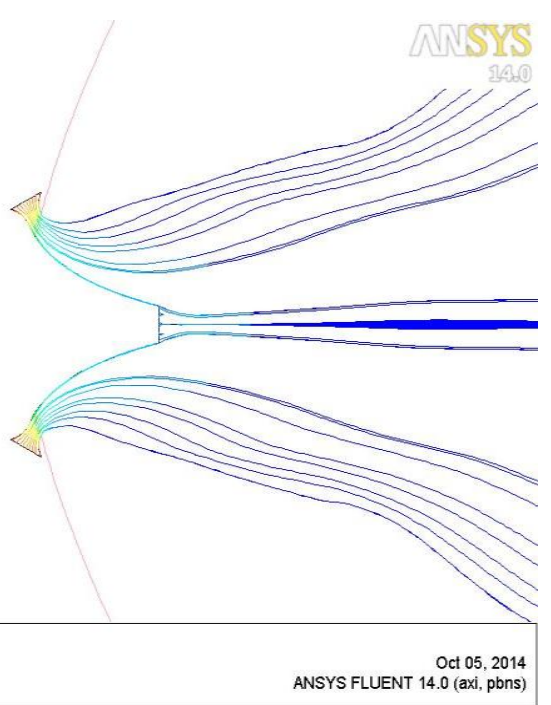

Oct 05, 2014
ANSYS FLUENT 14.0 (axi, pbns) 


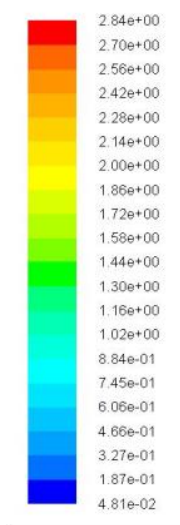

Contours of Mach Number

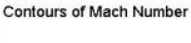

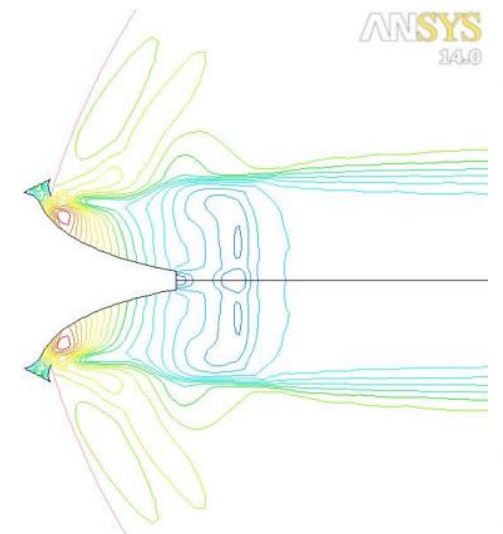
ANSYS FLUENT $\begin{array}{r}\text { OCt 05, } 2014 \\ 14.0 \text { (axi, pbns) }\end{array}$ Path

(e) Contours of Mach Number, $50 \%$ nozzle

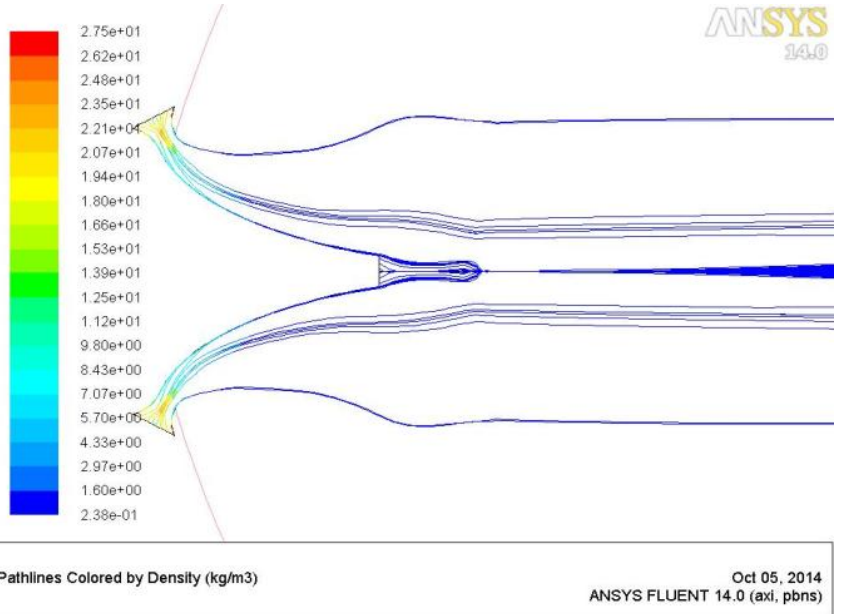

(f) Pathlines, 50\% nozzle

Fig-14: Flow pattern of the ideal and truncated aerospike nozzles in under-expansion conditions $($ Patm/Pdes $=0.10)$

\section{CONCLUSIONS}

The results clearly indicate that the aerospike nozzle is capable of producing the optimum performance at different altitudes.

From the simulation results we know that the base pressure compensates the loss of thrust in under-expansion conditions, plug truncation has minor effect on the loss of thrust in these conditions. But in over-expansion, thrust loss will increase with the increase of truncation. Base pressure thrust is closely related to variation of base pressure with atmospheric pressure. Base pressure is constant in underexpansion conditions, but increase with the increase of the atmospheric pressure in over-expansion conditions.

Based on the observed behavior of the exhaust flow, it can be concluded that the $40 \%$ truncated nozzle is recommended. Because its flow pattern shows the signs of optimum performance and it has achieved the desired exit Mach number in all the three altitude conditions.

\section{REFERENCES}

[1]. Angelino G., "Approximation Method for Plug Nozzle Design”, AIAA Journal, Vol. 2, No. 10, Oct. 1964, pp. 18341835 .

[2]. Gross, Klaus W., "Performance Analysis of Aerospike Rocket Engines," 1972.

[3] Lee, C. C., "Computation of plug nozzle contours by the Rao's optimum thrust method", NASA CR-21914 R-61, 1963.

[4]. Lee, C. C., Inman. S. J., "Numerical analysis of plug nozzles by the Method of characteristics", NASA TECHNICAL NOTE R-10, 1964.

[5]. Besnard, E., H. H. Chen, T. Mueller and J. Garvey, "Design, Manufacturing and Test of a Plug Nozzle Rocket Engine", AIAA Paper 2002-4038, 2002.

[6]. Naghib Lahouti, A., Nazarinia, M. and Tolouei, E., "Design and numerical analysis of aerospike nozzles with different plug shapes to compare their performance with a conventional nozzle", The Eleventh Australian International Aerospace Congress, Melbourne, Australia, 13-17 March (2005).

[7]. Tomita, T. et al., Nobuhiko, K. and Ogawara, A., "A conceptual system design study for a linear aerospike engine applied to a future SSTO Vehicle," The $46^{\text {th }}$ AIAA/ASME/SAE/ASEE Joint Propulsion Conference and Exhibit, AIAA-2010-7060, 2010.

[8]. Sakamoto, H., Takahashi M., Sasaki, M., Tomita, T., Kusaka K. and Tamura H., "An Experimental Study on a 14KN Linear Aerospike Nozzle Combustor", AIAA Paper 99- 2761, 1999.

[9]. Chang Hui Wang, Yu Liu, Li Zi Qin, "Aerospike nozzle contour design and its performance validation", Acta Astronautica 64 1264-1275, 2009.

\section{BIOGRAPHIES}

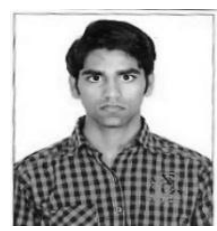

L. Vinay Kumar pursuing his M.Tech. in Aerospace Engineering from Aurora's Scientific and Technological Institute, Hyderabad, India. He did his B.tech from TKR College of Engineering, Hyderabad. His research interests include Computational Fluid Dynamics, H.V.A.C. Systems, cleanroom designing, Green building technologies.

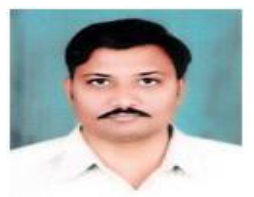

Dr.K.Srinivasa Reddy completed his B.E. in Mechanical Engineering with honours from NIT Silchar, Assam. He did his M.Tech. in IC Engines and Gas Turbines from NIT Warangal, Telegana. He was awarded with Ph.D. degree in Mechanical Engineering in the year 2011 by JNTUH, Kukatpally. He has 18 years of teaching experience and 2 years of $R \& D$ experience. His research interests include fluid dynamics, Heat Transfer and development of nonconventional energy utilisation technologies 